\title{
Conservación
}

\section{Los peces como indicador de restauración de áreas de manglar en la costa norte de Yucatán}

\author{
Fish as an indicator of ecological restoration of mangroves on the north coast of Yucatán \\ Daniel Arceo-Carranza ${ }^{\mathrm{a}, *}$, Eric Gamboa ${ }^{\mathrm{b}}$, Claudia Teutli-Hernández ${ }^{\mathrm{c}}$, Maribel Badillo-Alemán ${ }^{\mathrm{a}}$ \\ y Jorge Alfredo Herrera-Silveira ${ }^{\mathrm{d}}$ \\ a Laboratorio de Ecología, Unidad Multidisciplinaria de Docencia e Investigación, Unidad Sisal, Universidad Nacional Autónoma de México, \\ Puerto de Abrigo s/n, 97356 Sisal, Hunucmá, Yucatán, México

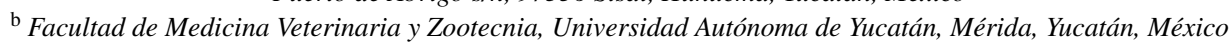 \\ c Departament d'Ecologia, Facultat de Biologia, Universitat de Barcelona, Av. Diagonal 643, E-08028, Barcelona, España \\ d Laboratorio de Producción Primaria, Centro de Investigación y de Estudios Avanzados del Instituto Politécnico Nacional, Unidad Mérida km 6, Antigua \\ Carretera Progreso, Cordemex, Mérida, Yucatán, México
}

Recibido el 3 de diciembre de 2014; aceptado el 11 de diciembre de 2015

Disponible en Internet el 16 de abril de 2016

\begin{abstract}
Resumen
Los manglares son reconocidos por los servicios ecológicos que proveen a muchas especies de peces. Estudios previos sobre restauración ecológica han pasado por alto estas funciones, generalmente enfocándose a la restauración de cobertura vegetal. El objetivo fue determinar las funciones de los manglares como zonas de refugio y alimentación para peces, evaluando la composición y abundancia en sitios con diferente tiempo de restauración. Se realizaron 2 muestreos en 4 sitios de manglar en restauración en Yucatán —Celestún 1, Celestún 2, Yucalpetén, Progreso-, en donde se registraron 22 especies de peces. Celestún 2 fue el sitio con mayor riqueza y diversidad (Gambusia yucatana, Poecilia velifera, Mugil cephalus y Anchoa mitchilli las más abundantes). Progreso registró la diversidad más baja y alta dominancia de G. yucatana (>90\%). El sitio con mayor tiempo de restauración presentó organismos juveniles detritófagos y zoobentófagos. Los resultados demuestran la función del manglar como zona de crianza y alimentación para especies marinas que intervienen en la transferencia de energía y nutrientes con sistemas adyacentes, también demuestran gran dominancia de G. yucatana, una especie endémica y característica del ecosistema de manglar en Yucatán.

Derechos Reservados (C) 2016 Universidad Nacional Autónoma de México, Instituto de Biología. Este es un artículo de acceso abierto distribuido bajo los términos de la Licencia Creative Commons CC BY-NC-ND 4.0.
\end{abstract}

Palabras clave: Restauración ecológica; Peces de manglar; Áreas de crianza; Funciones ecológicas; Celestún; Progreso

\section{Abstract}

Mangroves are well recognized for the ecological services that they provide to many fish species. Previous studies on ecological restoration have overlooked these ecological functions, as they are generally focused on the restoration of vegetation cover. The objective of this study is to determine the function of mangroves as nursery and feeding grounds for fishes, by assessing the species composition and abundance in sites with different restoration time. Two samplings were performed in 4 sites where mangrove is recovering along the Yucatán coast —Celestún 1, Celestún 2, Yucalpetén, Progreso- A total of 22 fish species were recorded, the higher richness and diversity were recorded in Celestún 2 (Gambusia yucatana, Poecilia velifera, Mugil cephalus and Anchoa mitchilli were the most abundant). In Progreso, the diversity was lower due to the dominance of G. yucatana $(>90 \%)$. The site with the longest restoration time presented juveniles of detritophagous and zoobentophagous species, which show the

\footnotetext{
* Autor para correspondencia.

Correo electrónico: darceo@ciencias.unam.mx (D. Arceo-Carranza).

La revisión por pares es responsabilidad de la Universidad Nacional Autónoma de México.
} 
nursery and feeding functions of the area. These marine species are involved in the transfer of energy and nutrients to adjacent systems, also a great dominance of G. yucatana was found, which is an endemic and characteristic fish species from the mangrove ecosystem in Yucatán.

All Rights Reserved (C) 2016 Universidad Nacional Autónoma de México, Instituto de Biología. This is an open access item distributed under the Creative Commons CC License BY-NC-ND 4.0.

\section{Introducción}

Las características estructurales y funcionales de los manglares brindan condiciones para que sean sitios de alimentación y reposo de aves migratorias; refugio de fauna silvestre; áreas de reproducción, alimentación y resguardo de muchas especies de peces e invertebrados (Beck et al., 2001; Nagelkerken et al., 2008; Wang, Huang, Shi y Wang, 2009). Además, se ha documentado que son excelentes almacenadores de carbono y exportadores de materia orgánica (Orihuela-Belmonte, TovillaHernández, Vester y Álvarez-Legorreta, 2004). En relación con la ictiofauna, Beck et al. (2001) definieron como hábitats de crianza aquellos sitios donde la producción de juveniles por área sea mayor que en otros hábitats. En distintas investigaciones se ha comparado la densidad de peces registrados en manglares de varias partes del mundo, en donde se ha estimado que 1 ha de manglar tiene el potencial para generar una producción secundaria entre 1 y 11.8 ton de peces/año/ha (Mirera, Kairo, Kimani y Waweru, 2010). Esta productividad puede ser explicada a partir de 2 hipótesis ecológicas: 1) la hipótesis de protección contra depredadores, que hace referencia a la complejidad estructural del hábitat que imponen los canales de marea, la turbidez, los neumatóforos y raíces de soporte, generando áreas de refugio a donde migran los peces aprovechando los ciclos de inundacióndesecación (Huxham, Kimani y Augley, 2004; Mirera et al., 2010; Shinnaka et al., 2007; Wang et al., 2009) y 2) hipótesis del manglar como zona de alimentación, la alta productividad biológica de estos ecosistemas ofrece gran cantidad de alimento en forma principalmente de detritus orgánico derivado de las hojas y raíces muertas (Huxham et al., 2004; Shinnaka et al., 2007; Wang et al., 2009).

Debido a la complejidad estructural de los manglares, el difícil acceso y a que es impráctico utilizar métodos convencionales de recolecta de peces como son redes de arrastre y chinchorros, son escasos los estudios que se enfocan a la recolecta entre los manglares, observándose más trabajos que comparan zonas de manglar con hábitats adyacentes como planicies lodosas o pastos sumergidos (Mirera et al., 2010; Shervette et al., 2007; Wang et al., 2009), así como con zonas de manglar deforestadas (Huxham et al., 2004; Mwandya, Gullström, Öhman, Andersson y Mgaya, 2009). La cobertura de manglares a nivel mundial está disminuyendo drásticamente (FAO, 2008). Cerca de 3,560 ha se perdieron entre 1980 y 2005. En México, el Inegi registra que para 1993 había 956,149 ha de manglar en las costas mexicanas, mientras que para el año 2000 se redujo la cobertura a 886,760 ha, alcanzando hasta un $7.8 \%$. Entre las principales causas que provocan esta degradación al ecosistema están la construcción de infraestructura turística, la sustitución por campos de cultivo y potreros, la camaronicultura, asentamientos humanos y la actividad petrolera (Herrera-Silveira y MoralesOjeda, 2010). Debido a esta acelerada pérdida de manglares, las autoridades de diversos países han puesto mayor atención llevando a cabo programas de restauración ecológica en los ecosistemas de manglar. En países como Colombia, Cuba y Panamá se realizan, principalmente, para mejorar la producción pesquera (Flores-Verdugo, Agraz-Hernández y Benítez-Pardo, 2006). En México, se están haciendo esfuerzos por parte de instituciones gubernamentales y no gubernamentales, específicamente en el estado de Yucatán para restaurar el ecosistema de manglar (Herrera-Silveira et al., 2012).

En la mayoría de los estudios sobre restauración ecológica, los indicadores de éxito se han relacionado principalmente con la respuesta en la cobertura y/o estructura vegetal, sin incluir indicadores del funcionamiento como medida del éxito de la restauración. Para el caso de la fauna, Macintosh, Ashton y Havanon (2002) mencionan que la recuperación de la biodiversidad ha sido uno de los principales indicadores utilizados para medir el éxito de las acciones de restauración. La diversidad de la ictiofauna en el ecosistema de manglar es una variable que define la salud del ecosistema (Velázquez-Velázquez y Vega-Cendejas, 2004), por lo tanto, y partiendo de que los manglares son sitios de alimentación y crianza de especies de peces e invertebrados, en este estudio el objetivo fue determinar la composición, abundancia y diversidad íctica en áreas de manglar sujetas a acciones de restauración ecológica.

\section{Materiales y métodos}

La valoración de la restauración del ecosistema de manglar se evaluó en 3 sitios localizados en la costa del estado de Yucatán: a) Progreso (fig. 1), el puerto más importante del norte de Yucatán, a $32 \mathrm{~km}$ de Mérida. En temporada veraniega presenta una intensa actividad industrial y turística. Las áreas de manglar han sido objeto de diversos impactos a causa de la apertura del puerto de abrigo: Yucalpetén; construcción de carreteras costeras, dragado de canales, entre otros. La zona de manglar muerto ( $>60$ ha) es claramente visible desde la carretera, la cual corta transversalmente el flujo laminar de agua, impidiendo el flujo superficial hacia la zona impactada. Presenta condiciones de hipersalinidad intersticial $(>120)$ y se mantiene inundada o sin agua superficial por largos periodos de tiempo. En la época de lluvias se observaban plántulas de Rhizophora mangle y Avicennia germinans, que desaparecían en la época de estiaje. Este sitio ha estado sujeto a diversas acciones de restauración ecológica 

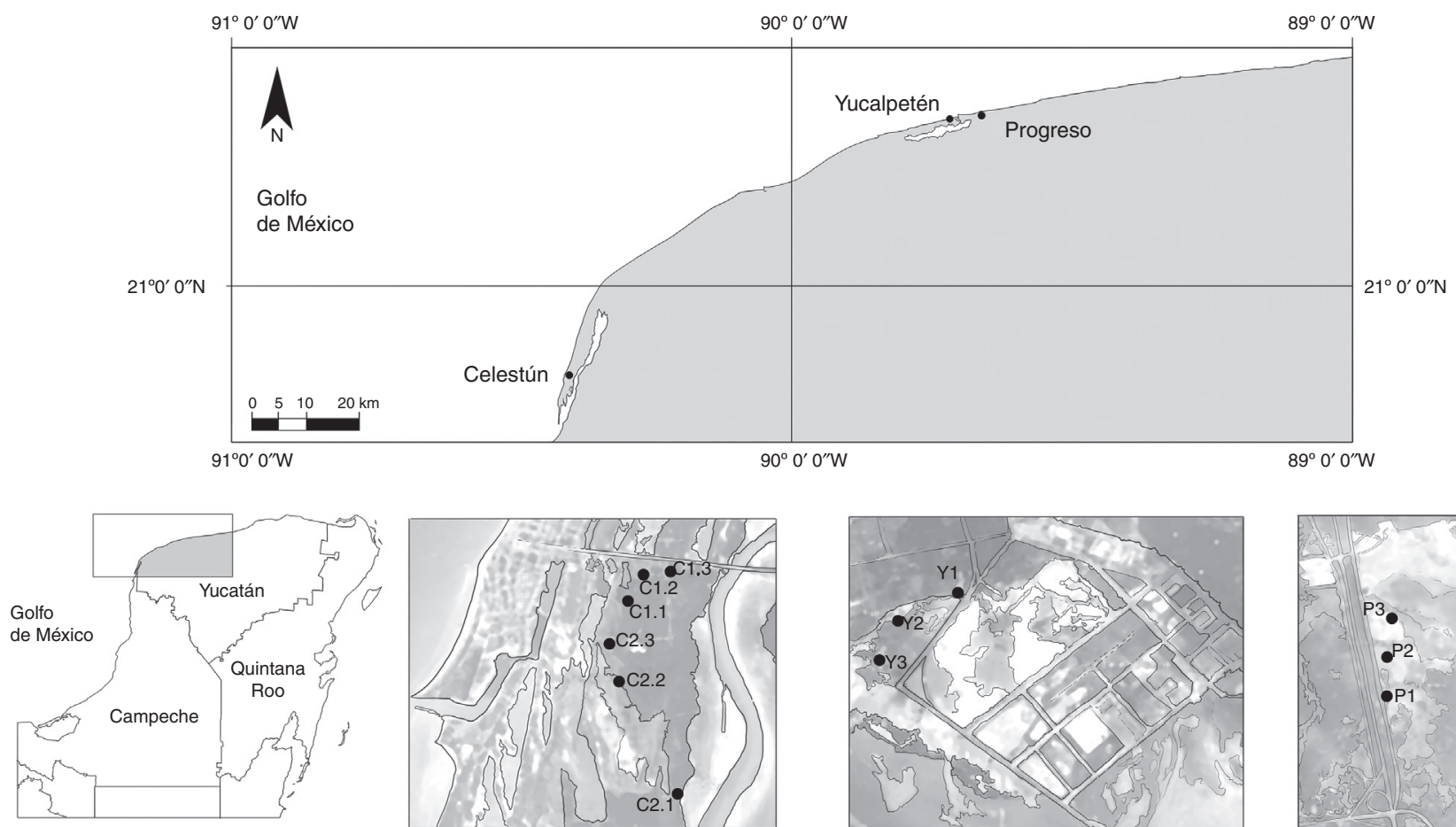

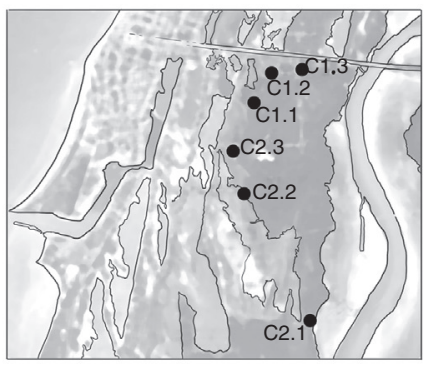

Celestún

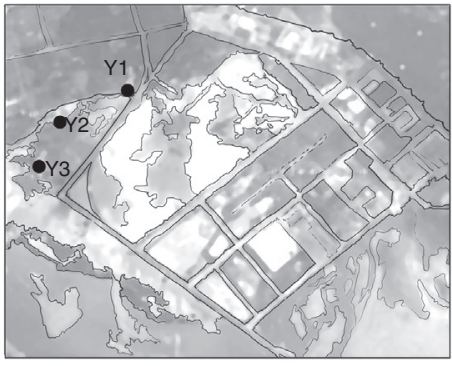

Yucalpetén

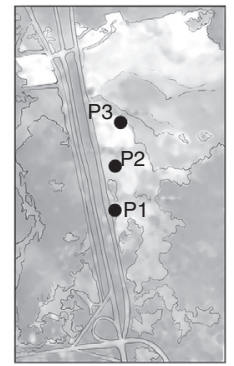

Progreso

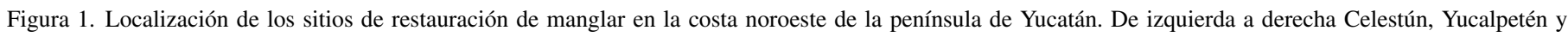
Progreso.

y de mantenimiento hidrológico desde 2009 (Echeverría-Ávila, 2013), actualmente se observan árboles $(1.50 \mathrm{~m})$ y plántulas de mangle negro, y algunas plántulas de mangle rojo, la salinidad intersticial es de 60 y los periodos de inundación están regulados temporalmente por acciones de marea y de la época de lluvias. b) Yucalpetén se localiza en el municipio de Progreso (fig. 1). Es una zona de manglar impactada por actividades antropogénicas, rellenada con sedimentos de dragado del puerto, lo cual modificó los factores hidrológicos que influyen sobre la estructura de la comunidad del bosque de manglar. Después de un tiempo de haber implementado acciones de restauración, se observa la dominancia de A. germinans con características de manglares chaparros, presencia de floración y alturas $<0.7 \mathrm{~m} \mathrm{y}$ crecimiento a lo ancho (Echeverría-Ávila, 2013). La rehabilitación del humedal por medio de la construcción de canales se inició en el año 2008. c) Celestún, a $85 \mathrm{~km}$ de la ciudad de Mérida (fig. 1), se caracteriza por el desarrollo pesquero y la actividad ecoturística de forma creciente desde los años 90 . En los años 70 se construyó la carretera que comunica Celestún con la ciudad de Mérida. Durante su construcción se interrumpieron los flujos de agua superficiales, iniciando la mortalidad masiva de manglares. La zona de manglar muerto (>100 ha) es claramente visible desde la carretera, la cual corta transversalmente microcuencas locales, impidiendo el flujo de agua superficial hacia la zona impactada. Antes de las acciones de restauración - construcción de canales- en el año 2005, en el sitio había salinidades intersticiales de 120 y superficiales $>100$, se mantenía inundado o seco por largos periodos de tiempo y solo se registraban troncos muertos sin la presencia de plántulas o semillas (Herrera-Silveira et al., 2012). Debido a que en este sitio de Celestún se construyeron 2 canales en diferentes años y cada zona presenta condiciones ambientales distintas, el sitio fue dividido en 2: 1) Celestún 1, que corresponde a un canal que se construyó en el 2007, con A. germinans y Laguncularia racemosa como especies dominantes después de la restauración hidrológica; 2) Celestún 2 ubicado entre la población del puerto de Celestún y la laguna, y que consiste de un canal hecho en 2006, con A. germinans como dominante. Esta zona de restauración se vio afectada por la construcción de una carretera y el paso de un huracán.

\section{Variables ambientales y recolecta de peces}

Se realizaron 2 recolectas en los 4 sitios de restauración: una en noviembre de 2011 y otra en marzo de 2012. En cada sitio se tomaron las muestras en los canales de restauración hidrológica, en donde cada canal se dividió equitativamente en 3 secciones, las cuales se consideraron réplicas. Se registraron in situ las variables hidrológicas de salinidad, oxígeno disuelto y temperatura con una sonda multiparamétrica YSI modelo 85 . Se recolectó sedimento superficial en los canales para la determinación de materia orgánica por el método de ignición (Davies, 1974).

La recolecta de peces se hizo con una red de arrastre modificada $(68 \times 70 \mathrm{~cm}, 1 \mathrm{~mm}$ de luz de malla), realizando 2 arrastres con longitud de barrido de $6.8 \mathrm{~m}^{2}$. Los ejemplares capturados se colocaron en hielo y transportaron al laboratorio en donde se fijaron con formol al $4 \%$ y se mantuvieron en alcohol al $70 \%$. Los peces se identificaron hasta nivel específico con ayuda de claves especializadas (Greenfield y Thomerson, 1997; Miller, 

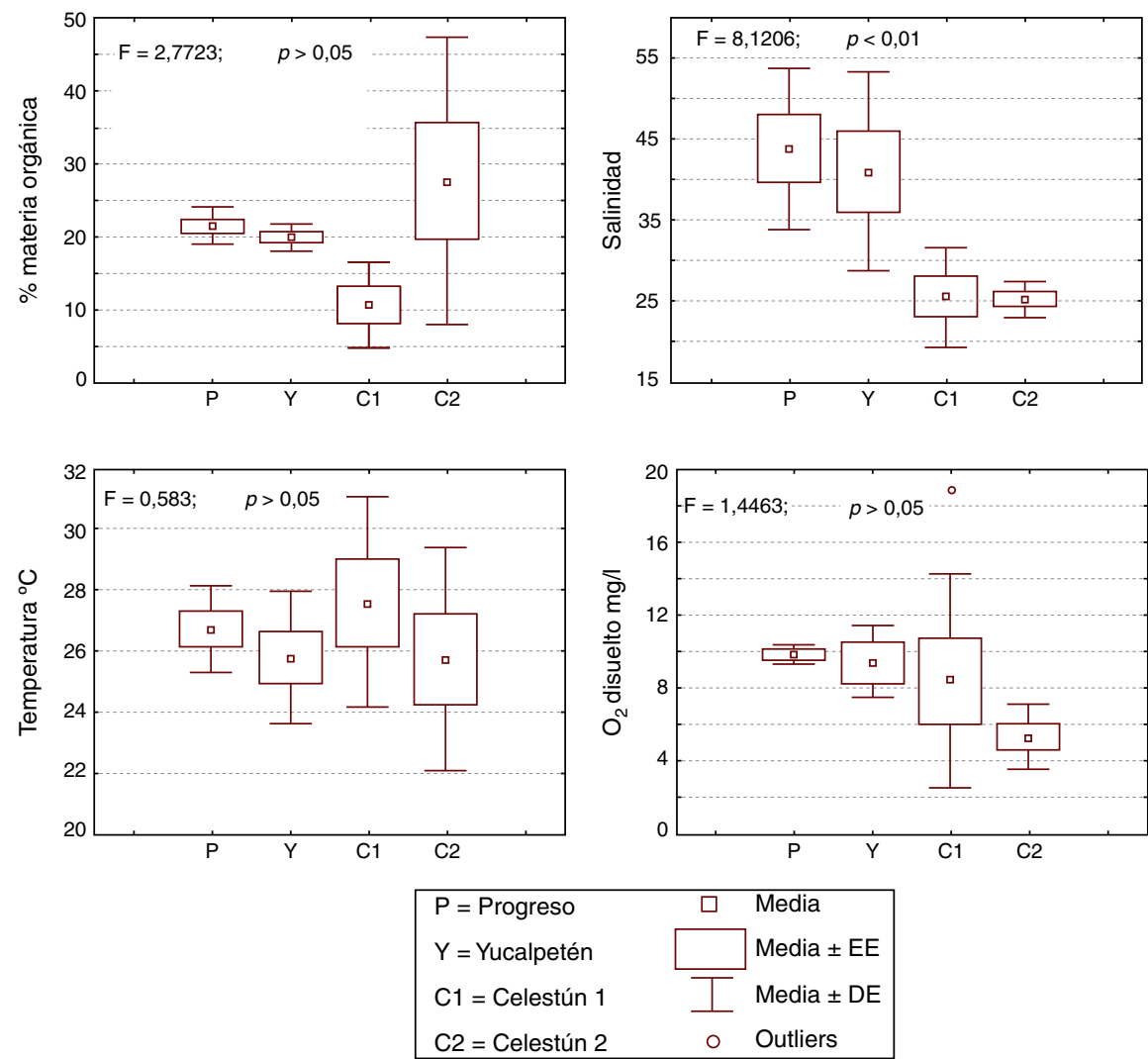

Figura 2. Variación y diferencias de materia orgánica, salinidad, temperatura y oxígeno disuelto entre sitios de muestreo; se presentan los resultados del análisis de varianza (F y p).

2009; Schmitter-Soto, 1998), y se registraron las medidas morfométricas de talla en LE $(\mathrm{cm})$ y peso $(\mathrm{g})$, y se determinó de acuerdo con información de la literatura (Froese y Pauly, 2013) si son especies en estadio juvenil o adulto.

\section{Análisis de datos}

Para caracterizar la ictiofauna de los sitios de restauración, se estimaron los parámetros comunitarios de diversidad (ShannonWiener), equidad (Pielou), abundancia y riqueza de especies con el programa Primer 6. Las diferencias entre las variables hidrológicas de los sitios — materia orgánica, salinidad, temperatura y oxígeno disuelto- en diferente grado de restauración, se evaluaron por medio de un análisis de varianza (ANOVA) comprobando previamente que se cumplieran los supuestos de normalidad y homogeneidad de varianza a través de las pruebas de Lilliefors (Kolmogorov-Smirnov modificado) $(\mathrm{p}>0.05)$ y Bartlett ( $p>0.05)$, respectivamente.

Para comparar estadísticamente entre los sitios de restauración de manglar y determinar diferencias en la composición íctica, se realizó un análisis de contraste de hipótesis «analysis of similarities» (ANOSIM) y, posteriormente pruebas a posteriori para detectar entre qué pares de muestras hay diferencias. Asimismo, para determinar la asociación entre sitios de restauración de acuerdo con la composición íctica, se utilizó un análisis de clasificación y un análisis MDS basado en la matriz de similitud de Bray-Curtis con el programa Primer 6 (Clarke y Gorley, 2006).

\section{Resultados}

Las variables hidrológicas mostraron diferencias en relación con la salinidad $\mathrm{p}<0.01$, siendo los sitios de Progreso y Yucalpetén los que presentaron condiciones hiperhalinas, mientras que los de Celestún mostraron menores salinidades; la temperatura, oxígeno disuelto y porcentaje de materia orgánica no mostraron diferencias entre sitios (fig. 2).

Se registraron 22 especies en total. En cuanto al número de especies por sitio, se registraron 11 en Celestún 1, 12 en Progreso y 13 especies en Yucalpetén y Celestún 2. La abundancia de especies fue muy variable, la menor en Celestún 2 -289 individuos-, y la mayor en Yucalpetén —2,097— (tabla 1). De acuerdo con las tallas recolectadas se determinó que el total de las especies marinas son organismos en estadio juvenil. Se utilizaron índices de diversidad y de equidad como indicadores de la restauración, en donde el sitio que presentó la mayor diversidad (1.6 bits/ind) y mayor equidad (0.65) fue Celestún 2 (fig. 3).

Tabla 1

Número de especies de peces (S) y de organismos (N) en los sitios de recolecta.

\begin{tabular}{llr}
\hline Sitio & $\mathrm{S}$ & $\mathrm{N}$ \\
\hline Progreso & 12 & 1,731 \\
Yucalpetén & 13 & 2,097 \\
Celestún 1 & 11 & 1,913 \\
Celestún 2 & 13 & 289 \\
\hline
\end{tabular}


Tabla 2

Especies dominantes e indicadoras de restauración de las funciones de crianza (C) y de alimentación (A), categoría ecológica $-^{1}$ dulceacuícola; ${ }^{2}$ Marinos estenohalinos-y tiempo de restauración de los sitios de estudio.

\begin{tabular}{|c|c|c|c|}
\hline Sitio & Especies dominantes & Especies indicadoras & $\begin{array}{l}\text { Tiempo de restauración } \\
\text { (año de inicio) }\end{array}$ \\
\hline Progreso & G. yucatana ${ }^{1}$ & B. belizanus ${ }^{1}$ (A) & 2 años (2010) \\
\hline Yucalpetén & $\begin{array}{l}\text { G. yucatana } 1 \\
\text { P. velifera }{ }^{1}\end{array}$ & $\begin{array}{l}\text { M. } \text { cephalus }^{2}(\mathrm{~A}, \mathrm{C}) \\
\text { M. trichodon } \\
\text { E. } \text { harengulus }^{2}(\mathrm{C}, \mathrm{C})\end{array}$ & 3 años (2009) \\
\hline Celestún 1 & $\begin{array}{l}\text { G. yucatana }{ }^{1} \\
\text { M. } \text { cephalus }^{2}\end{array}$ & $\begin{array}{l}\text { A. } \text { michilli }^{2}(\mathrm{C}) \\
\text { M. } \text { cephalus }^{2}(\mathrm{~A}, \mathrm{C}) \\
\text { B. belizanus } \\
1\end{array}$ & 6 años (2006) \\
\hline Celestún 2 & $\begin{array}{l}\text { G. yucatana } \\
\text { P. velifera }{ }^{1} \\
\text { M. } \text { cephalus }^{2} \\
\text { A. michilli }\end{array}$ & $\begin{array}{l}\text { A. } \text { michilli }^{2}(\mathrm{C}) \\
\text { M. cephalus }^{2}(\mathrm{~A}, \mathrm{C}) \\
\text { B. chrysoura } \\
\text { S. testudineus }^{2}(\mathrm{C}) \\
\text { E. gula }^{2}(\mathrm{C}) \\
\text { E. harengulus }^{2}(\mathrm{C})\end{array}$ & 8 años (2004) \\
\hline
\end{tabular}

La abundancia relativa por especie muestra la dominancia de Gambusia yucatana en más del $75 \%$ en Progreso, Yucalpetén y Celestún 1 (fig. 4). El sitio con mayor tiempo de restauración fue Celestún 2, el cual presenta una dominancia compartida por más especies (tabla 2). Gambusia yucatana fue la especie dominante por su abundancia en los 4 sitios de restauración. Se observa que esta dominancia es menor conforme aumenta el tiempo de restauración -Progreso, Yucalpetén, Celestún 1 y Celestún 2 (fig. 4) - , y que la riqueza de especies se incrementa conforme al tiempo en que las acciones de restauración ecológica se llevaron a cabo. En Celestún 2, el sitio con mayor tiempo de restauración, se registraron más especies (tabla 2).

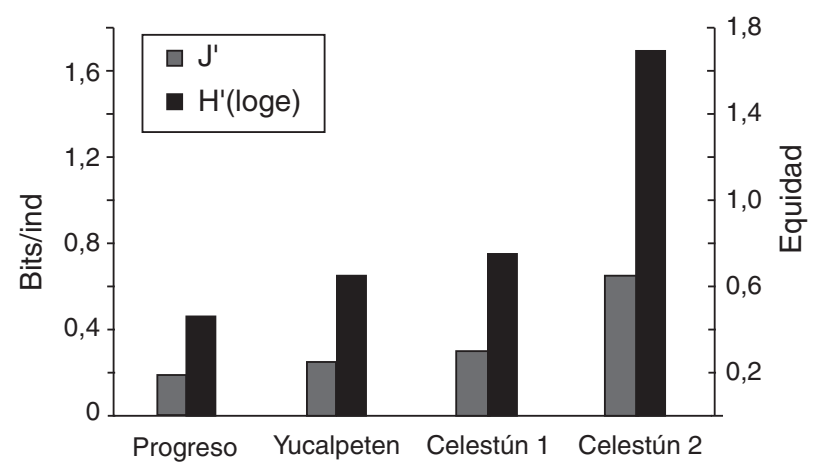

Figura 3. Parámetros comunitarios de las especies ícticas (diversidad y equidad) en los sitios de restauración de manglar.

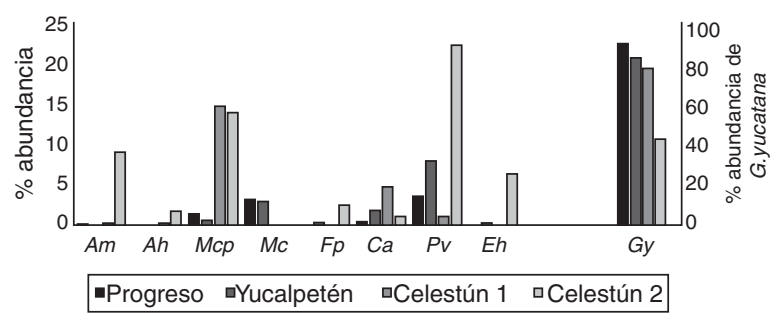

Figura 4. Abundancia relativa de las especies ícticas en los sitios de estudio. Ah: Anchoa hepsetus; Am: Anchoa mitchilli; Ca: Cyprinodon artifrons; Eh: Eucinostomus harengulus; Fp: Floridichthys polyommus; Gy: Gambusia yucatana; Mc: Menidia colei; Mcp: Mugil cephalus; Pv: Poecilia velifera.
Tabla 3

Resultados de las pruebas post hoc del análisis de similitudes (ANOSIM), se muestra el factor sitio, el valor del estadístico $\mathrm{R}$ y el valor de significación $(p)$, los resultados menores a 0.05 están marcados $(* *)$.

\begin{tabular}{lll}
\hline Sitios & $\mathrm{R}$ & Nivel de significación $(p)$ \\
\hline Progreso-Yucalpetén & 0.143 & 0.119 \\
Progreso-Celestún 1 & 0.076 & 0.173 \\
Progreso-Celestún 2 & 0.15 & 0.110 \\
Yucalpetén-Celestún 1 & 0.309 & $0.011^{* *}$ \\
Yucalpetén-Celestún 2 & 0.33 & $0.019 * *$ \\
Celestún 1-Celestún 2 & 0.267 & $0.022 * *$ \\
\hline
\end{tabular}

El análisis de similitud (ANOSIM) indicó diferencias significativas entre sitios $(\mathrm{p}<0.05)$, al realizarse las pruebas pareadas se identificaron los sitios de restauración que mostraron dichas diferencias (tabla 3), principalmente Celestún 2 con Yucalpetén y con Celestún 1, así como Celestún 1 con Yucalpetén. El análisis MDS generado conjuntamente con el análisis de clasificación de conglomerados muestra la formación de 2 grupos a un nivel del $60 \%$ de similitud. Uno conformado por la asociación de los sitios Progreso, Yucalpetén y Celestún 1, y el segundo considerando Celestún 2 como un grupo independiente (fig. 5).

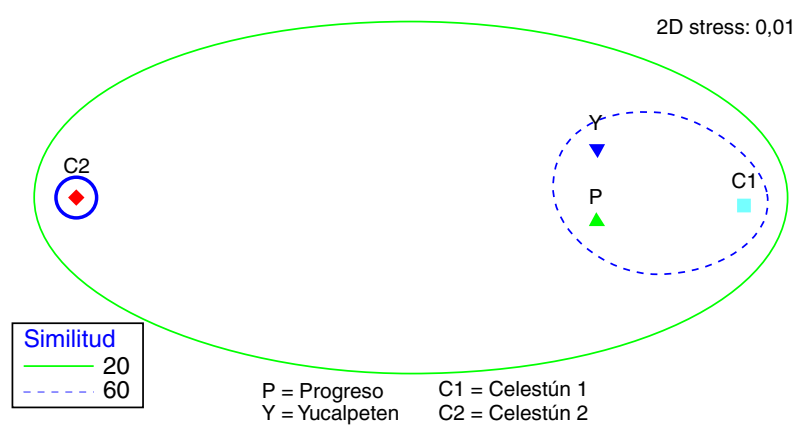

Figura 5. Análisis MDS que muestra el agrupamiento de los sitios de restauración con base en la similitud de la composición de especies. 


\section{Discusión}

Se presentaron diferencias significativas de la salinidad entre sitios de restauración, siendo mayor en Progreso y Yucalpetén (>40), y menor en Celestún 1 y Celestún $2(<25)$. Esto puede deberse a los afloramientos subterráneos que aportan agua dulce a la zona de los canales y que se ve reflejado en una disminución de la salinidad en los canales (25.19), mientras que en la laguna de Chelem la salinidad promedio es mayor (40.91) (Tapia-González, Herrera-Silveira y Aguirre-Macedo, 2008). Este factor es importante para los peces marinos eurihalinos que habitan las zonas costeras, incluyendo los manglares. CastroAguirre, Espinoza-Pérez y Schmitter-Soto (1999) clasifican a los peces del golfo de México de acuerdo con su tolerancia a la salinidad, así como Whitfield, Taylor, Fox y Cyrus (2006) mencionan que los peces marinos eurihalinos son los más abundantes en salinidades entre 10 y 40.

La laguna de Chelem es el principal aporte de agua para los canales de las zonas de restauración de Progreso y Yucalpetén. En esta laguna se han registrado 53 especies de peces (Vargas y Vega-Cendejas, 2010), pero solamente 18 de ellas en los sitios de restauración. Las especies registradas en este estudio pertenecen al grupo que utiliza los manglares como áreas de refugio, crecimiento y alimentación, ya que se observó alta dominancia de juveniles, mostrando que este sitio de manglar en restauración tiene un papel importante como hábitat de crianza (Beck et al., 2001). Este resultado indica que las acciones de restauración están favoreciendo la recuperación de funciones ecológicas, y con esto potenciales servicios ambientales. En Celestún, VegaCendejas (2004) registra 48 especies que utilizan los manglares de esta zona, la mayoría marinas eurihalinas con dependencia de hábitats estuarinos para crecer y alimentarse. En los 2 canales de restauración de Celestún se registraron 16 especies que ya han sido documentadas por Vega-Cendejas (2004) para los manglares de Celestún. Destacan organismos juveniles de Anchoa mitchilli, Anchoa hepsetus, Mugil curema, M. cephalus, Bairdiella chrysoura, Sphoeroides testudineus, Eucinostomus gula y E. harengulus, las cuales tienen comportamiento migratorio entre manglares y el mar, confirmando el papel funcional del ecosistema de manglar.

La especie más abundante en todas las zonas de restauración fue G. yucatana (Poecilidae). Especie endémica del norte de la península de Yucatán que habita normalmente zonas de canales y aguas someras cercanas a los manglares, con hábitos omnívoroplanctófagos (Mondragón-Sánchez y Rodas-Regil, 2011). Esta especie tiene una importante función en estos ecosistemas al constituir un recurso alimenticio para peces de mayor tamaño y como regulador de las poblaciones de otros organismos acuáticos como son larvas de insectos (Mondragón-Sánchez y RodasRegil, 2011). Otra especie de la misma familia (Poecilia velifera) es la segunda con mayor abundancia en los sistemas costeros de la península de Yucatán. Esta especie es común en ambientes dulceacuícolas y salobres, es omnívora, pero puede explotar recursos como zoobentos o materia de origen vegetal a manera de detritus (Froese y Pauly, 2013). Es importante en el flujo energético entre sistemas, ya que es presa de peces que migran de sistemas adyacentes, y aves que anidan en los manglares (Ramo y Busto, 1993; De Dios, 2014). Adicionalmente se encuentra incluida en la Nom-059 por su estatus como especie endémica.

En los sitios de estudio se registraron peces de tamaño pequeño (0.1-0.5 cm LE), de especies marinas, las cuales han sido registradas como dominantes en las asociaciones pezmanglar (Bosire et al., 2008). Los resultados robustecen la hipótesis de que la restauración ecológica favorece la recuperación de funciones de hábitats críticos, ya que en este caso se ha favorecido la crianza de peces de especies marinas que utilizan los manglares en etapas juveniles de su ciclo de vida; estos resultados han sido documentadas por otros autores (Laegdsgaard y Johnson, 2001; Maci y Basset, 2009; Wang et al., 2009). En relación con Fundulus persimilis registrada en Progreso, se ha documentado que utiliza el hábitat de las raíces de manglar para actividades reproductivas en diferentes localidades de la península de Yucatán (Arceo-Carranza y Vega-Cendejas, 2009), indicando que la restauración ecológica de manglares está siendo exitosa no solo desde el punto de vista de la recuperación de estructura vegetal (Herrera-Silveira et al., 2010), sino que se están recuperando funciones ecológicas importantes que podrían llevar a restablecer servicios ecológicos de provisión y sostén.

Respecto a la hipótesis de que la restauración de manglares ofrecerá posibilidades de alimentación a especies, existen peces detritívoros que se alimentan de esta producción generada por los manglares; dentro de ellos encontramos a los mugílidos. La presencia de organismos juveniles de M. cephalus (1-4.6 cm LE) muestra que la restauración de los canales de marea del manglar en Celestún está favoreciendo que este hábitat sea utilizado como área de alimentación y crecimiento para especies de origen marino. Se ha observado que juveniles de la familia Mugilidae no consumen invertebrados como los adultos, sino que se alimentan de fango y materia orgánica (Franco y Bashirullah, 1992), y ambos son componentes dominantes en los canales de marea restaurados.

En cuanto a los depredadores tope, en Progreso y Celestún 1, la presencia del pez ictiófago Belonesox belizanus muestra que las condiciones de estos hábitats se están recuperando favorablemente, ya que estas especies son indicadoras de interacciones tróficas de diferentes niveles, e indican una comunidad saludable con estructuras tróficas diversificadas (Velázquez-Velázquez y Vega-Cendejas, 2004).

La composición y abundancia de la ictiofauna en manglares con diferente tiempo de restauración indica que, a través del tiempo, la estructura comunitaria es más diversa. En el sitio de Celestún 2 con 10 años de restauración ecológica, se presenta una estructura comunitaria de peces más compleja al incluir especies de distintos niveles tróficos, además de que la presencia de organismos juveniles de especies marinas (E. gula, E. harengulus, S. testudineus, B. chrysoura, A. mitchilli y M. cephalus) sugiere que el sitio restaurado está funcionando como área de refugio. Esto puede observarse en el análisis multidimensional escalado (fig. 5), ya que el sitio de Celestún 2 (60\% de similitud) se encuentra como grupo independiente y Celestún 1, Progreso y Yucalpetén forman un grupo debido a la similitud que presentan en la composición de especies. Por otra parte, Progreso es el sitio que presenta la menor estructura comunitaria de peces; en este sitio la abundancia y dominancia de G. yucatana es de más del 
90\% — especie omnívora, tolerante a ambientes degradados-, no se presentan especies marinas y es el sitio con riqueza específica, diversidad y equidad más bajos, esto considerando que es el sitio con menor tiempo de restauración hidrológica.

El éxito de la restauración de manglares ha sido evaluado principalmente a través de la recuperación de la estructura y cobertura vegetal. Sin embargo, la evaluación de recuperación de las funciones como hábitat crítico de especies endémicas, de importancia comercial y para la conservación de la biodiversidad, ha sido escasamente valorada. Los resultados de este estudio indican que es probable que las funciones ecológicas de un ecosistema de manglar restaurado se recuperen más rápidamente que las de la vegetación, por la rápida disponibilidad de refugio y alimentación que representa la rehabilitación de los canales de marea de manglar.

Por otra parte, los tiempos de restauración ecológica en los bosques de manglar del presente estudio muestran recuperación de la complejidad de los ensamblajes de peces. La restauración ecológica de estos manglares en Yucatán está restableciendo funciones ecológicas como la biodiversidad y producción secundaria que no habían sido demostradas con anterioridad en esta región del golfo de México. La restauración de funciones ecológicas como sitios de alimentación y crianza determinando los parámetros comunitarios, la presencia de peces juveniles y los gremios tróficos nos permiten determinar el estado de perturbación de una comunidad. Con respecto a las funciones ecológicas que brinda el manglar, los indicadores propuestos para el caso de Celestún y Progreso permitieron hacer una primera evaluación al estado de restauración en el ecosistema de manglar.

\section{Agradecimientos}

El presente estudio se realizó con el apoyo del proyecto «Estudio ecológico regional para el fortalecimiento de un programa de conservación y rehabilitación de humedales de manglar en la península de Yucatán». Fonsec-Conafor-Conacyt, S0002-71529, y con el Programa de Apoyo a Proyectos de Investigación e Innovación Tecnológica (PAPIIT-DGAPA) con el proyecto IA202913 «Los manglares como zonas de refugio de peces. Comparación entre zonas restauradas en el norte de la península de Yucatán». Agradecemos al M. en C. Alfredo Gallardo-Torres su ayuda en la identificación ictiológica.

\section{Referencias}

Arceo-Carranza, D. y Vega-Cendejas, M. E. (2009). Spatial and temporal characterization of fish assemblages in a tropical coastal system influenced by freshwater inputs: Northwestern Yucatán peninsula. Revista de Biología Tropical, 57, 89-103.

Beck, M. W., Heck, K., Jr., Able, K. W., Childers, D. L., Eggleston, D. B., Gillanders, B. M., et al. (2001). The identification, conservation, and management of estuarine and marine nurseries for fish and invertebrates. BioScience, 51 633-641.

Bosire, J. O., Dahdouh-Guebas, F., Walton, M., Crona, B. I., Lewis, R. R. III, Field, C., et al. (2008). Functionality of restored mangroves: a review. Aquatic Botany, 89, 251-259.

Castro-Aguirre, J. L., Espinoza-Pérez, H. S. y Schmitter-Soto, J. J. (1999). Ictiofauna estuarino lagunar y vicaria de México. Colección textos politécnicos. Serie Biotecnologías. México, D.F.: Editorial Limusa.
Clarke, K. R. y Gorley, R. (2006). Primer V6: User manual/tutorial. Plymouth, United, Kingdom: Primer-E.

Davies, B. (1974). Loss-on ignition as an estimate of soil organic matter. Soil Science Proceedings, 38, 150.

De Dios, A. C. (2014). Ecología trófica de un gremio de aves piscívoras durante la temporada de reproducción en la costa de Yucatán Tesis de maestría. Posgrado de Ciencias del Mar y Limnología. Universidad Nacional Autónoma de México.

Echeverría-Ávila, S. C. (2013). Evaluación del crecimiento de Avicennia germinans en un sitio de restauración ecológica en el puerto de Progreso, Yucatán Tesis. Universidad Autónoma de Yucatán.

FAO (Food and Agriculture Organization). (2008). La desaparición de manglares alcanza un nivel alarmante. Recuperado 19 Jun 2014 de: http:/www.fao.org/Newsroom/es/news/2008/1000776/index.html

Flores-Verdugo, F. J., Agraz-Hernández, C. M. y Benítez-Pardo, D. (2006). Creación y restauración de ecosistemas de manglar: principios básicos. En P. Moreno-Casasola, E. Peresbarbosa y A. C. Travieso-Bello (Eds.), Manejo integral de la zona costera: un enfoque municipal. Vol. II (pp. 10931110). Xalapa, Veracruz: Instituto de Ecologia, A.C. y Comisión Nacional de Áreas Naturales Protegidas (Secretaría de Medio Ambiente y Recursos Naturales).

Franco, L. y Bashirullah, M. B. (1992). Alimentación de la lisa (Mugil curema) del golfo de Cariaco-Estado Suche, Venezuela. Zootecnia Tropical, 10, 219-238.

Froese, R. y Pauly, D. (Eds.). (2013). FishBase. World Wide Web Electronic Publication. Recuperado 30 Oct 2013 de: www. fishbase.org

Greenfield, D. W. y Thomerson, J. E. (1997). Fishes of the continental waters of Belize. Florida: University Press of Florida.

Herrera-Silveira, J. A. y Morales-Ojeda, S. (2010). Lagunas costeras. En R. Durán y M. Méndez (Eds.), Biodiversidad y desarrollo humano en Yucatán. Mérida: CICY, PPD-FMAM, Conabio, Seduma.

Herrera-Silveira, J. A., Teutli-Hernández, C., Zaldivar-Jiménez, M., RiveraMonroy, V., Coronado-Molina, C., Hernández-Zaavedra, R., et al. (2010). Marco conceptual de la rehabilitación de bosques de manglar en la península de Yucatán (SE, México). En Manual práctico para la rehabilitación del ecosistema de manglar en Yucatán, México (Tercera edición). Mérida, Yucatán: JICA-Conanp. Proyecto de conservación de humedales en la península de Yucatán.

Herrera-Silveira, J. A., Zaldivar-Jiménez, A., Teutli-Hernández, C., PérezCeballos, R., Caamal, J. y Andueza, T. (2012). Rehabilitación de manglares en el estado de Yucatán sometidos a diferentes condiciones hidrológicas y nivel de impacto: el caso de Celestún y Progreso. México, D.F.: Centro de Investigación y de Estudios Avanzados, Unidad Mérida. Informe final SNIB-Conabio. Proyecto GH009.

Huxham, M., Kimani, E. y Augley, J. (2004). Mangrove fish: a comparison of community structure between forested and cleared habitats. Estuarine Coastal and Shelf Science, 60, 637-647.

Laegdsgaard, P. y Johnson, C. R. (2001). Why do juvenile fish utilize mangrove habitats? Journal of Experimental Marine Biology and Ecology, 257, 229-253.

Maci, S. y Basset, A. (2009). Composition, structural characteristics and temporal patterns of fish assemblages in non-tidal Mediterranean lagoons: a case study. Estuarine Coastal and Shelf Science, 83, 602-612.

Macintosh, D. J., Ashton, E. C. y Havanon, S. (2002). Mangrove rehabilitation and intertidal biodiversity: a study in the Ranong mangrove ecosystem Thailand. Estuarine Coastal and Shelf Science, 55, 331-345.

Miller, R. R. (2009). Peces dulceacuícolas de México. México, D.F: Conabio, Simac, Ecosur, Desert Fish Council.

Mirera, D., Kairo, J. G., Kimani, E. y Waweru, F. (2010). A comparison between fish assemblages in mangrove forests and on intertidal flats at Ungwana Bay, Kenya. African Journal of Aquatic Science, 35, 165-171.

Mondragón-Sánchez, A. y Rodas-Regil, O. (2011). Hábitos alimentarios de Gambusia yucatana. Kuxulkab’ Revista de Divulgación. División Académica de Ciencias Biológicas, XVII, 43-48.

Mwandya, A. W., Gullström, M., Öhman, M. C., Andersson, M. H. y Mgaya, Y. (2009). Fish assemblages in Tanzanian mangrove creek systems influenced by solar salt farm constructions. Estuarine Coastal and Shelf Science, 82, 193-200. 
Nagelkerken, I., Balber, S., Bouillon, S., Green, P., Haywood, M., Kirton, L. G., et al. (2008). The habitat function of mangroves for terrestrial and marina fauna: a review. Aquatic Botany, 89, 155-185.

Orihuela-Belmonte, E., Tovilla-Hernández, C., Vester, H. F. y ÁlvarezLegorreta, T. (2004). Flujo de materia en un manglar de la costa de Chiapas, México. Madera y Bosques, 45, 61.

Ramo, C. y Busto, B. (1993). Resources use by herons in a Yucatán wetland during the breeding seasons. Wilson Bulletin, 105, 573-586.

Schmitter-Soto, J. J. (1998). Catálogo de los peces continentales de Quintana Roo. San Cristóbal de las Casas: El Colegio de la Frontera Sur.

Shervette, V. R., Aguirre, W. E., Blacio, E., Cevallos, R., González, M., Pozo, F., et al. (2007). Fish communities of a disturbed mangrove wetland and an adjacent tidal river in Palmar, Ecuador. Estuarine Coastal and Shelf Science, $72,115-128$

Shinnaka, T., Sano, M., Ikejima, K., Tongnunui, P., Horinouchi, M. y Kurokura, H. (2007). Effects of mangrove deforestation on fish assemblage at Pak Phanang Bay, southern Thailand. Fisheries Science, 73, 862-870.

Tapia-González, F. U., Herrera-Silveira, J. A. y Aguirre-Macedo, M. L. (2008). Water quality variability and eutrophic trends in karstic tropical coastal lagoons of the Yucatán Peninsula. Estuarine Coastal and Shelf Science, 76 , 418-430.

Vargas, M. K. E. y Vega-Cendejas, M. E. (2010). Composición y distribución íctica en el estero de Yucalpetén. En R. Durán y M. Méndez (Eds.), Biodiversidad y desarrollo humano en Yucatán (p. 496). Mérida: CICY, PPD-FMAM, Conabio, Seduma.

Vega-Cendejas, M. E. (2004). Ictiofauna de la Reserva de la Biosfera Celestún, Yucatán: una contribución al conocimiento de su biodiversidad. Anales del Instituto de Biología de la Universidad Nacional Autónoma de México. Serie Zoológica, 75, 193-206.

Velázquez-Velázquez, E. y Vega-Cendejas, M. E. (2004). Los peces como indicadores del estado de salud de los sistemas acuáticos. CONABIO. Biodiversitas 57, 12-15.

Wang, M., Huang, Z., Shi, F. y Wang, W. (2009). Are vegetated areas of mangroves attractive to juvenile and small fish? Estuarine Coastal and Shelf Science, 85, 208-216.

Whitfield, A. K., Taylor, R. H., Fox, C. y Cyrus, D. P. (2006). Fishes and salinities in the St. Lucia estuarine system-a review. Reviews in Fish Biology and Fisheries, 1, 1-20. 